\title{
Ant-Based Systems for Wireless Networks: Retrospect and Prospects
}

\author{
Laurent Paquereau ${ }^{1}$ and Bjarne E. Helvik ${ }^{2}$ \\ 1 Department of Telematics, \\ Norwegian University of Science and Technology, Trondheim, Norway \\ laurent@item.ntnu.no \\ 2 Centre for Quantifiable Quality of Service in Communication Systems ${ }^{\star}$, \\ Norwegian University of Science and Technology, Trondheim, Norway \\ bjarne@q2s.ntnu.no
}

\begin{abstract}
Since they were first introduced as powerful stochastic optimization systems, ant-based systems have been applied to a wide range of problems. One of the most successful applications has been routing in dynamic wired telecommunication networks. Following this success, similar approaches have been applied to routing in multi-hop wireless networks. The objective has been to achieve self-organizing path management in these networks. This paper looks back on 10 years of research and presents reflections on the challenges, the evolution, the contributions and the future perspectives in this field.
\end{abstract}

\section{Introduction}

Ant-based systems are systems inspired by the foraging behavior of ants in nature or, more generally, systems that can be described as such. Ant-based systems are swarm intelligence systems. Each individual ant is not capable of finding the shortest path between its nest and a food source. It is by their collective behavior that ants in a colony are able to converge to the shortest path; a key enabler to this emergent behavior being the indirect communication between ants through the modification of their environment (stigmergy).

Ant-based systems were first developed as powerful stochastic optimization systems and shown to be able to achieve state-of-the-art results on complex combinatorial problems. Later, ant-based techniques were combined with the idea of using mobile agents for path management in next-generation telecommunication networks. The motivation was to apply stochastic optimization in the network and develop decentralized, adaptive, robust and self-organizing solutions to cope with the growing complexity of networks. On the basis of the promising results obtained for wired networks in the second half of the 1990's, ant-based systems for routing in multi-hop wireless networks (MHWNs) started to be developed, the first of them being ARA [16] proposed in 2002.

\footnotetext{
* "Centre for Quantifiable Quality of Service in Communication Systems, Centre of Excellence" appointed by The Research Council of Norway, funded by the Research Council, NTNU and UNINETT. http://www.q2s.ntnu.no
} 
In the context, ants are control packets that are used to repeatedly sample paths between source and destination pairs. At each node, the pheromone trails reflect the knowledge acquired by the colony, and the pheromone trail value associated with a given neighbour indicates the relative goodness of this neighbour to reach a specific destination.

The objective with this paper is neither to present yet another extensive survey nor a detailed description of the inner working of ant-based systems for MHWNs. For that, the interested reader is referred to [1518]. Rather, it is to look back on 10 years of research on applying ant-based techniques to routing in MHWNs and present some reflections on the challenges, evolutions, contributions, trends and perspectives in the field, touching upon both technical and non-technical aspects.

The rest of this paper is organized as follows. First, Section 2 addresses the application of ant-based techniques to mobile ad-hoc networks (MANETs), which is the most common type of MHWNs ant-based techniques have been applied to. Next, Section 3 discusses the evolution and current status of the research in the field and the perspectives for future developments and applications. Finally, concluding remarks are given in Section 4 .

\section{Applying Ant-Based Techniques to MANETs}

This section addresses the application of ant-based techniques to MANETs. The focus is on unicast routing protocols as this represents the major part of the contributions, but there have also been proposals for using ant-based techniques for establishing and maintaining multicast trees in the context of MANETs, e.g. MANSI [23].

This section elaborates first on the premises for developing ant-based systems for routing in MANETs and the challenges related to the application of antbased techniques in this context. The main solutions found in the literature are then presented. Finally, all these elements are summarized and discussed.

\section{$2.1 \quad$ Premises}

MANETs are autonomous MHWNs in which nodes communicate without the support of any pre-existing infrastructure and with no centralized control. Nodes may operate both as routers and hosts. They are all potentially mobile and battery-powered, and they may have limited processing and memory capacity. MANETs typically exhibit a high degree of dynamicity as nodes may move and leave or join the network at any time, making control, and in particular routing, a challenging task. Moreover, in addition to being able to deal with the changes in the environment, any control system designed for MANETs has to cope with the limited bandwith and the inherent unreliability of wireless communications, making this task even more challenging.

The main reasons that are put forward in the literature to support the application of ant-based techniques to routing in MANETs are: (i) the good match 
between the desirable properties of a path management systems for MANETs and the characteristics of ant-based systems in terms of adaptivity and robustness, and, as mentioned in the introduction, (ii) the promising results obtained applying ant-based techniques to routing in wired networks, in particular with AntNet [10]. Adaptivity is the ability of the system to adapt to changes and prevent disruptive events. This feature is empowered in ant-based systems by the repeated stochastic sampling of end-to-end paths and the resulting update of pheromone trail values at each node. It ensures the availability of timely routing information and enables the discovery and maintenance of multiple paths between source and destination pairs. Robustness is the ability of the system to maintain persitent operation under the occurrence of perturbations. Typical perturbations in MANETs include the loss of packets and the loss of routing information stored at nodes. Ant-based systems are robust in that they are not sensitive to the loss of individual ants and that routing information (pheromone trail values) is distributed throughout the network.

\subsection{Challenges}

The main challenge when applying ant-based techniques to routing in MANETs is efficiency. This is a two-sided challenge. Efficiency can be expressed in terms of routing overhead and in terms of convergence time. Others challenges include the proactive nature of ant-based systems, spreading the load, demands in terms of memory and processing and energy consumption.

Routing Overhead. The routing overhead for ant-based systems is related to the repeated probing of paths realized by ants. This implies forwarding control packets back and forth between source and destination pairs which does consume bandwidth. As the wireless medium is shared and the available bandwidth is limited, controlling the overhead is therefore crucial for the performance of the system (data delivery ratio, delay, delay jitter). However, there is a trade-off between performance on the one side, and adaptivity and robustness on the other side. Ant-based systems are intrinsically robust to the loss of individual ants because they generate many of them. Reducing the number of ants directly affects the robustness of the system. In addition, the ability of the system to discover new paths and adapt to topological changes is conditioned by the fact that ants sample paths and update pheromone tables accordingly. Reducing the number of ants also directly affects the adaptivity of the system.

Convergence Time. Ant-based systems apply stochastic optimization and several iterations are required before such a system converges to a solution. Iterations corresponds to updates made by ants. Hence, the time it takes for a system to converge is related to the rate at which ants are generated. To be able to adapt, the time scale at which changes take place in the network should be much larger than that at which pheromone trail values are updated. Since MANETs are assumed to be highly dynamic environments and the number of ants required to keep up with the changes may be prohibitive with respect to the routing overhead incurred. 
Proactive Nature. Ant-based systems rely on the proactive sampling of paths and comsume resources forwarding ants and maintaining pheromone tables at each node. In a wired networks, this makes sense as the topology is seldom changing, and, although nodes or links might failed, it is expected that they are restored with the same characteristics so that previously gathered data are still relevant and can actually be used to re-converge faster. In the context of MANETs, spending resources for proactive planning does generally not pay off as the conditions, in terms of topology, wireless link characteristics and traffic demands, are constantly changing. In addition, keeping historical data may be harmful for performance. When a node loses connectivity with one of its neighbor, although connectivity might be re-established at a later point in time with the same node, there is no guarantee that the quality of the path through this neighbor will be similar. Previously collected data may no longer be relevant and may lead to reduced performance if used.

Load Spreading. The promising results obtained with AntNet in wired networks are in part due to its ability to achieve near-optimal load distribution by spreading the load along multiple path proportionally to their estimated quality. Achieving similar results in the context of MANETs is challenging for several reasons: (i) efficiently spreading the load in a highly dynamic environment is difficult, (ii) the behavior of the nodes and the traffic patterns in MANETs are unpredictable hence no assumptions that would help in distributing the load can be made, and (iii) distributing the load across multiple paths in MHWNs may lead to degraded performance because of interferences if the paths are not radio-disjoint [12 20].

Memory, Processing and Energy Constraints. Ant-based systems rely on the repeated and stochastic sampling of paths by ants and the maintenance of pheromone tables locally at each node. At each node, the pheromone table potentially contains an entry for each destination in the network through each of the neighbouring nodes. This implies repeated computations on a per-packet basis and imposes requirements in terms of processing and storage at each node. In addition, performing these operations and regularly forwarding ants are energy consuming tasks.

\subsection{Solutions}

Ant-based systems developed for MANETs are mostly derived from AntNet or directly apply the Ant Colony Optimization (ACO) meta-heuristic [11. Systems vary among others in terms of formulas used for pheromone updates, cost functions (hop-count, delay, or some measure of link quality including signalto-noise ratio, congestion or packet loss ratio, or any combination of those) and mechanisms used to improve the performance. Studies such as 39] have shown that directly applying ant-based systems developed for wired networks such as AntNet results in lower performance compared to conventional MANETs 
protocols, and in particular to AODV [21, which is used as the reference protocols in most of the studies. In the following, the focus is on the mechanisms introduced specifically to address the challenges listed in Section 2.2

Broadcasting Forward Ants. Most of the systems broadcast forward ants to explore multiple routes concurrently and, hence, reduce the convergence time. However, this approach has also several downsides: (i) paths are sampled regardless of their estimated quality, (ii) it results in a large overhead as ants get duplicated at each node, (iii) it results in the establishment of paths with low quality, and (iv) it leads to the discovery and reinforcement of a set of braided (interfering) paths that cannot therefore not be used to improve the performance of system by distributing the load. The resulting process is similar to the route discovery process of AODV, and some systems also introduce mechanisms used by AODV to limit the overhead such as expanding ring-search. To limit the overhead other systems such as AntHocNet [13] resolve to broadcast only when no routing information is available at a node and otherwise apply a random proportional rule and unicast the ant only to one of the neighboring nodes.

Adopting a Greedy Deterministic Forwarding Policy. Most of the systems abandon the stochastic forwarding policy used in AntNet for distributing the load accross multiple path and instead adopt a greedy deterministic forwarding policy, i.e. each node forwards data packets to its neighbor with the highest pheromone concentration for the given destination. The main reasons, as mentioned above, are that it is difficult to distribute the load in a dynamic environment and that distributing the load might lead to degraded performance because of interferences. Authors in 12 also show that the best performance with AntHocNet is achieved when a greedy deterministic policy is applied to the forwarding of ants. The reason is that the number of ants that can be generated is often too low for the system to be able to cope with the constantly changing environment, and the system cannot effectively explore multiple paths.

Relying on Data Packets for Updating Pheromone Values. In order to reduce the overhead, some of the proposed systems rely partly or solely on data packets to update pheromones, e.g. ARA and SARA [6]. The major drawback of this approach is that it affects directly the adaptivity and robustness of the system as it becomes dependent on data traffic to maintain timely routing information. Using data packets for exploration also results in decreased performance in terms for instance of increased delay and jitter.

Combining Reactive and Proactive Path Sampling. To reduce the overhead, most of the proposed systems adopt a reactive strategy, meaning that a node starts generating ants only when it is itself source of data traffic. Many systems keep, however, a proactive component and are therefore classified as hybrid, e.g. AntHocNet. The information collected by proactive ants may then used for bootstraping the reactive route discovery process and/or for improving the path and adapting to changes while data sessions are active. 
Introducing Additional Control Packets for Link Error Notification and Handling. To improve the adaptivity of the system, many systems introduce additional dedicated control packets for explicit link failure notifications, local recovery and/or to deal with lost link failure notifications, e.g. ARA and AntHocNet. One disadvantage of these mechanisms is that they create an extra overhead upon link failure. Another aspect is that local recovery techniques are opposite with the objective of converging towards a global optimum by constructing complete solutions, which is one of the advantages of ant-based systems as a stochastic optimization method.

Introducing Pheromone Diffusion. This mechanism is introduced in AntHocNet. The idea is to let nodes share routing information with their 1-hop neighborhood via asynchronous beacon messages and derive virtual pheromones that are used to guide the exploration of ants.

\subsection{Discussion}

To deal with the challenges listed in Section 2.2 and bear the comparison with state-of-the-art reactive MANET routing protocols, in particular AODV, most of the proposed systems include mechanisms borrowed from those same protocols. This introduces a deviation from key elements of the optimization method (proactive sampling of end-to-end solutions, stochastic forwarding policy) and from what made its successful application to dynamic routing problem in wired networks (load-spreading). As a result, most of the systems are intermediate between conventional reactive MANET routing protocols and ant-based systems developed for path management in wired networks, such as AntNet. Some of them are much closer to AODV although the terminology used is that of ant-based systems.

We argue that ant-based techniques have been applied to MANETs on wrong premises and question the suitability of this approach for routing in MANETs in the first place. The adaptability and robustness properties of an ant-based systems stem from the repeated sampling of path by ants, but are conditioned by the fact that the intensity of changes the system has to adapt to is sufficiently low compared to the rate at which ants sample paths and update pheromone trails. In addition, there is a trade-off between how adaptive and robust an antbased system can be and the routing overhead it generates. In typically settings for MANETs, changes occur too fast for the overhead not to be prohibitive.

\section{Evolution, Status and Perspectives}

In this section, we first present the evolution and the current status of the research on ant-based techniques for wireless networks. We then discuss when this approach is viable and when it is no, the question of the future of this approach and sketch possible future directions. 


\subsection{Evolution}

The development of the first generation of ant-based systems for MHWNs, targeting MANETs, started at the beginning of the 2000's; the first ant-based system for MANETs reported in the literature being ARA [16]. The explanation of why it started at that time is that: (i) routing in MANETs was a hot topic in the second half of the 1990's and early in 2000, and that, concurrently, (ii) promising results achieved by ant-based systems for wired networks were published by artificial intelligence researchers and attracted the attention of the MANET research community. Reversely, the development of ant-based systems for MANETs by networking researchers later attracted the attention of the artificial intelligence community (AntHocNet was first reported in 2004). Another reason is (iii) the observation made by De Couto et al. in 2002 [8] that minimizing the number of hops to decide on the best route between nodes in a MHWN is not necessarily synonym of maximizing the performance as longer links are generally more unstable than shorther ones. The fact that it is possible to integrate the link/path quality in the computation of the pheromone trail values is listed as an argument for applying ant-based techniques to MANETs in [16].

Starting from 2004, and following the trend observed in MHWN research, a second generation of ant-based systems, targeting specialized MHWNs, started to be developed. The reason for this specialization is that the definition of MANETs is very generic and encompasses a wide range of scenarios and applications, which cannot all be best solved by a single protocol design. In particular, ant-based systems were developed for Wireless Sensor Networks (WSNs) [2] and backbone Wireless Mesh Networks (WMNs) [1]. The characteristics of these specialized MHWNs and of generic MANETs are summarized in Table 1 .

Table 1. Characteristics of MHWNs

\begin{tabular}{|c||c|c|c|c|c|c|}
\hline MHWNs & $\begin{array}{c}\text { Limited } \\
\text { power, } \\
\text { memory, } \\
\text { processing }\end{array}$ & Mobility & $\begin{array}{c}\text { Predictable } \\
\text { traffic } \\
\text { patterns }\end{array}$ & $\begin{array}{c}\text { High rate } \\
\text { of changes } \\
\text { in the } \\
\text { network }\end{array}$ & $\begin{array}{c}\text { Limited } \\
\text { bandwidth }\end{array}$ & $\begin{array}{c}\text { Inter- } \\
\text { ferences }\end{array}$ \\
\hline \hline MANET & $\mathrm{X}$ & $\mathrm{X}$ & & $\mathrm{X}$ & $\mathrm{X}$ & $\mathrm{X}$ \\
\hline \hline $\mathrm{WSN}$ & $\mathrm{X}$ & & $\mathrm{X}$ & & $\mathrm{X}$ & $\mathrm{X}$ \\
\hline \hline $\mathrm{WMN}$ & & & $\mathrm{X}$ & & $\mathrm{X}$ & $\mathrm{X}$ \\
\hline
\end{tabular}

In particular, a relatively large number of ant-based systems targeting WSNs have been designed, see for instance [22] for a survey. WSNs have characteristics that make proactive approaches and load spreading a priori more appropriate than in generic MANETs: (i) nodes are static and, (ii) certain traffic patterns are expected between sensor nodes and sink nodes making load distribution both easier and more relevant. Compared to generic MANETs, restrictions in terms of memory and processing capacity as well as energy constraints are however more compelling. Hence, one can also question the intrinsic suitability of ant-based approaches in this context. 
Similarly to WSNs, nodes in WMNs are static and the traffic is expected to be concentrated on certain paths (to and from gateway nodes providing connection to the Internet). On the other hand, contrary to WSNs, nodes in WMNs are gridpowered wireless routers with high processing and memory capacity. Therefore, although the number of contributions addressing specifically backbone WMNs is still limited, they seem to be the most actual type of MHWNs for the application of ant-based techniques. An example of such a system is for instance AMIRA [4].

Finally, it should be noted that the research in the field has mainly been be driven by method-oriented rather than problem-oriented researchers. As for wired networks with AntNet, the most significant work in the field to date, AntHocNet, has been contributed by authors with a background in artifical intelligence. Applying ant-based techniques to MHWNs was, in a way, the next step after the promising results achieved with AntNet.

\section{$3.2 \quad$ Status}

As we assess the state of art, the research in ant-based techniques for routing in MANETs is about to cease, because: (i) as concluded in Section 2, ant-based systems are intrinsically not suitable for MANETs, and (ii) although several of the proposed systems and in particular AntHocNet are shown to outperform AODV, the gain in performance is not worth the added complexity. This latter assertion is supported by the work of AntHocNet authors who first observed in 12 that stochastically sampling paths results in lower performance compared to deterministically resampling the best paths, and who later proposed a new approach for integrating proactive and reactive routing in MANETs that built up on observations they made with AntHocNet, but with no references to ant-based techniques 14 .

The application of ant-based techniques to specialized MHWNs is, on the other hand, still an open research topic. In spite of the fact that one may question its suitability for WSNs, many such systems have been proposed. It is, however, hard to assess the performance these proposals, since comparisons with state-of-the-art algorithms specifically designed for WSNs are seldom provided. Furthermore, both real-world implementations and testing, as well as mathematical modeling, is also listed as missing in [22].

In the context of WMNs, few ant-based systems have been proposed so far. Pursuing these further, a first necessary step is a comparison with conventional state-of-the-art proactive routing protocols such as OLSR [17]. In the context of MANETs, ant-based systems have most of the time only been compared with AODV. To the best of our knowledge, no comparisons with traditional proactive MANET routing protocols have been reported.

\subsection{Perspectives}

Have ant-based techniques in MHWNs a future? If we expect it to be a simple, efficient and generally applicable technique, the answer is no. The technological 
rationale for this is found in Section 2. The method has hereto not been able to overcome the significant challenges put by the highly dynamical, wireless domain. On the other hand, we argue that the path management problem in WMNs is closer to that addressed in wired network than that addressed in MANETs, and that thus ant-based systems may have a future in this context. Moreover, there are specific aspects in the path management problem in WMNs for which antbased systems are relevant and attractive candidates. For instance, mesh routers are usually assumed to be equiped with multiple wireless interfaces and the channel assignment problem presents some similarities with the graph coloring problem for which good results have been reported using ant-based systems in a static context [7].

Although the path management problems in WMNs is closer to those of wired network than those addressed in MANETs, the challenges related to wireless communication - limited bandwidth and interferences - remain. Hence, applying systems developed for wired networks directly to WMNs leads to poor performance. One observation is that to rectify the problems incurred in the wireless domain, the typical approach has been to fall back on conventional path finding methods and patch ant-based systems with extra mechanisms, rather than devising new approaches. As an alternative direction for future research, we suggest to instead adapt the execution of the ant-based primitives to the context of wireless networks. One such approach is OAS [19. The idea is to apply the opportunitic forwarding paradigm [5] to the forwarding of ant agents. The fundamental changes are to defer the stochastic forwarding decision until after the reception of an ant by the potential next-hop nodes, and to execute it in a distributed manner among the receivers and in the time-domain rather than at the sender node and in the space-domain. This yields a reduction of the overhead and improves the adaptivity of the system compared to its unicast counterpart. Preliminary experiments indicate that such a system is able to achieve to efficiently spread the load along multiple paths [20].

\section{Concluding Remarks}

In this paper, we have retrospectively looked at 10 years of research in the field of ant-based systems for wireless networks. We have questionned the suitability of the approach for generic MANETs as well as for WSNs. We have presented the evolution and the current status of the research in this field and sketched a possible future direction of research for WMNs.

The suitability issue may also be raised for ant-based path management of dynamic telecommunication networks in general. In our view, the future might be in what we term ant-assisted systems rather than in ant-based systems. Rather than being the sole mean for self-organization, ant-based techniques seem more suitable as an element of a coumpound system using an array of techniques. In such a system, ants are not used for the primary path management task, but instead as a background process for the optimization of the primary system in the long run, e.g. the tuning of internal parameters, the detection of failure 
situations or degraded performance, or the optimization of external parameters affecting the path management problem. Applying ant-based techniques to the long-term optimization of the channel assignment in WMNs without relying on ants to solve the routing problem itself would for instance fall into this category.

\section{References}

1. Akyildiz, I., Wang, X., Wang, W.: Wireless mesh networks: a survey. Computer Networks 47(4), 445-487 (2005)

2. Akyildiz, I., Su, W., Sankarasubramaniam, Y., Cayirci, E.: Wireless sensor networks: a survey. Computer Networks 38(4), 393-422 (2002)

3. Baras, J.S., Mehta, H.: A probabilistic emergent routing algorithm for mobile ad hoc networks. In: 1st International Symposium on Modeling and Optimization in Mobile, Ad Hoc, and Wireless Networks, WiOpt (2003)

4. Bokhari, F., Zaruba, G.: AMIRA: interference-aware routing using ant colony optimization in wireless mesh networks. In: IEEE Wireless Communications and Networking Conference, WCNC (2009)

5. Bruno, R., Nurchis, M.: Survey on diversity-based routing in wireless mesh networks: Challenges and solutions. Computer Communications 33(3), 269-282 (2010)

6. Correia, F., Vazão, T.: Simple ant routing algorithm strategies for a (multipurpose) MANET model. Ad Hoc Networks 8(8), 810-823 (2010)

7. Costa, D., Hertz, A.: Ants can colour graphs. Journal of the Operational Research Society 48(3), 295-305 (1997)

8. De Couto, D., Aguayo, D., Chambers, B., Morris, R.: Performance of multihop wireless networks: Shortest path is not enough. In: 1st Workshop on Hot Topics in Networks (HotNets), ACM SIGCOMM (2002)

9. Dhillon, S.S., Arbona, X., Van Mieghem, P.: Ant routing in mobile ad hoc networks. In: 3rd IEEE International Conference on Networking and Services, ICNS (2007)

10. Di Caro, G.A., Dorigo, M.: AntNet: Distributed Stigmergetic Control for Communications Networks. Journal of Artificial Intelligence Research 9, 317-365 (1998)

11. Dorigo, M., Di Caro, G.A., Gambardella, L.M.: Ant algorithms for discrete optimization. Artificial Life 5(2), 137-172 (1999)

12. Ducatelle, F., Di Caro, G.A., Gambardella, L.M.: An Analysis of the Different Components of the AntHocNet Routing Algorithm. In: Dorigo, M., Gambardella, L.M., Birattari, M., Martinoli, A., Poli, R., Stützle, T. (eds.) ANTS 2006. LNCS, vol. 4150, pp. 37-48. Springer, Heidelberg (2006)

13. Ducatelle, F.: Adaptive Routing in Ad Hoc Wireless Multi-hop Networks. Ph.D. thesis, University of Lugano, Switzerland (2007)

14. Ducatelle, F., Di Caro, G.A., Gambardella, L.M.: A new approach for integrating proactive and reactive routing in MANETs. In: 5th IEEE International Conference on Mobile Ad Hoc and Sensor Systems, MASS (2008)

15. Ducatelle, F., Di Caro, G.A., Gambardella, L.M.: Principles and applications of swarm intelligence for adaptive routing in telecommunications networks. Swarm Intelligence 4(3), 173-198 (2010)

16. Günes, M., Sorges, U., Bouazizi, I.: ARA-the ant-colony based routing algorithm for MANETs. In: International Workshop on Ad Hoc Networking, IWAHN (2002)

17. Jacquet, P., Muhlethaler, P., Clausen, T., Laouiti, A., Qayyum, A., Viennot, L.: Optimized link state routing protocol for ad hoc networks. In: IEEE International Multi Topic Conference, INMIC (2001) 
18. Farooq, M., Di Caro, G.: Routing protocols for next-generation networks inspired by collective behaviors of insect societies: An overview. In: Blum, C., Merkle, D. (eds.) Swarm Intelligence: Introduction and Applications. Natural Computing Series, pp. 101-160. Springer, Heidelberg (2008)

19. Paquereau, L., Helvik, B.E.: Opportunistic Ant-Based Path Management for Wireless Mesh Networks. In: Dorigo, M., Birattari, M., Di Caro, G.A., Doursat, R., Engelbrecht, A.P., Floreano, D., Gambardella, L.M., Groß, R., Şahin, E., Sayama, H., Stützle, T. (eds.) ANTS 2010. LNCS, vol. 6234, pp. 480-487. Springer, Heidelberg (2010)

20. Paquereau, L., Helvik, B.E.: Ant-Based Multipath Routing for Wireless Mesh Networks. In: Di Chio, C., Brabazon, A., Di Caro, G.A., Drechsler, R., Farooq, M., Grahl, J., Greenfield, G., Prins, C., Romero, J., Squillero, G., Tarantino, E., Tettamanzi, A.G.B., Urquhart, N., Uyar, A.Ş. (eds.) EvoApplications 2011, Part II. LNCS, vol. 6625, pp. 31-40. Springer, Heidelberg (2011)

21. Perkins, C., Royer, E.: Ad-hoc on-demand distance vector routing. In: 2nd IEEE Workshop on Mobile Computing and Applications (1999)

22. Saleem, M., Di Caro, G.A., Farooq, M.: A review of swarm intelligence based routing protocols for wireless sensor networks. Information Sciences 181(20), 4597-4624 (2011)

23. Shen, C.C., Jaikaeo, C.: Ad hoc multicast routing algorithm with swarm intelligence. Mobile Networks and Applications 10(1), 47-59 (2005) 